\title{
Commissioning and Performance of the ATLAS Transition Radiation Tracker with Cosmic Rays and First High Energy Collisions
}

\author{
Peter Wagner \\ University of Pennsylvania, Philadelphia, USA
}

on behalf of the ATLAS Collaboration

\begin{abstract}
The Transition Radiation Tracker is the outermost of the three subsystems in the ATLAS Inner Detector. It contributes significantly to the precision of the momentum measurement of charged particles and to the identification of electrons. This note reports about the recent commissioning progress and the performance achieved in preparation for first collisions at the LHC.
\end{abstract}

Key words: Commissioning, Cosmics, Collisions, ATLAS, TRT

PACS: X, Y

\section{Introduction and Design}

The Transition Radiation Tracker (TRT) [1] is the outermost subsystem of the inner detector at ATLAS [2] which is im- ${ }_{35}$ 4 mersed in a $2 \mathrm{~T}$ solenoid field. The TRT is a straw drift-tube ${ }_{36}$ detector operated in proportional mode with a total of $350848_{37}$ readout channels. It contributes significantly to the precision of the momentum measurement of charged particles and to the ${ }_{39}$ identification of electrons. As a continuous tracker it provides a large number $(\sim 30)$ of measurement points ("straw hits") on track with a hit resolution of $130 \mu \mathrm{m}$. The straw drift tubes are 40 made of thin Kapton-based multilayer material. They have a diameter of $4 \mathrm{~mm}$ and contain a $30 \mu \mathrm{m}$ diameter, gold-plated ${ }^{41}$ tungsten anode wire in the center. The small tube radius limits ${ }^{42}$ the maximum drift time to $\sim 50 \mathrm{~ns}$. The length of the straws ${ }^{43}$ has been chosen such that the counting rate per wire is not ${ }^{44}$ more than $\sim 20 \mathrm{MHz}$ at LHC design luminosity. The straw ${ }^{45}$ wall, which lies at a potential of $-1.5 \mathrm{kV}$ relative to the wire, ${ }^{46}$ provides a separation between the transition radiation medium ${ }^{47}$ and the active gas $\left(70 \% \mathrm{Xe}, 27 \% \mathrm{CO}_{2}, 3 \% \mathrm{O}_{2}\right)$. To minimize ${ }^{48}$ the number of radiation lengths only light materials were used ${ }^{49}$ throughout the detector.

When a charged particle traverses a straw it typically loses a ${ }^{51}$ ${ }_{23}$ few hundred eV energy to ionization clusters of electronc and ${ }^{52}$ 24 ions in the gas. The liberated electrons drift towards the wire, ${ }^{53}$ where the high electric field leads to secondary ionizations in ${ }^{54}$ the gas with an average gain of 25000 . This signal is modi- ${ }^{55}$ fied with an analog Amplifier Shaper Discriminator Baseline ${ }^{56}$ Restorer ("ASDBLR") where it is discriminated on a $\sim 300 \mathrm{eV}^{57}$ threshold. To permit a hit resolution of $130 \mu \mathrm{m}$, the signal is ${ }^{58}$ then recorded in a digital pipeline of $3.125 \mathrm{~ns}$ wide time bins ${ }^{59}$ in a Digital Time Measurement Read-Out Chip ("DTMROC") 60

Email address: peter. wagner@cern.ch (Peter Wagner) before it is sent to the back-end electronics. The DTMROC can further (a) configure the ASDBLR to inject a test pulse used to estimate the effects of radiation damage, (b) sense the low voltage applied to both ASDBLR and DTMROC, (c) transmit configuration and sense data to the trigger, timing and control (TTC) back-end during data taking used for e.g. single event upset recovery, and (d) transmit a trigger signal to the TTC back-end (see Section 5).

\section{Tracking}

The recorded signals from the anode wires provide information about both the particle tracks and the synchronization in time of the various readout channels. To discriminate between noise and straw signals from particles, the energy deposited in the straw is required to be higher than a "tracking threshold" of $300 \mathrm{eV}$. The trailing edge of the discriminated signal from a particle is caused by the latest electrons in the avalanche that come from close to the straw wall. The timing of the trailing edge can be tuned in groups of 200 straws on the front-end boards such that all readout signals fit well within the $75 \mathrm{~ns}$ readout window. Figure 1 shows the relative readout timing $\left(t_{0}\right)$ distribution of all barrel and endcap boards separately, using data from Fall 2009 where single LHC beam bunches were shot on closed LHC collimators $\sim 140$ m upstream of ATLAS ("beam splashes"). The timing spread in the barrel has been measured to be within $1 \mathrm{~ns}$ for more than $97 \%$ of the readout channels. The outliers in these histograms have already been adjusted as of this writing. Note that timing variations are corrected on a finer granularity separately in calibrations for offline track reconstruction.

The leading edge of the straw signal comes from avalanche electrons closest to the wire and is correlated to the distance that these electrons drift from track to wire, and hence to the track to wire distance. Figure 2 shows, using LHC collision data, 


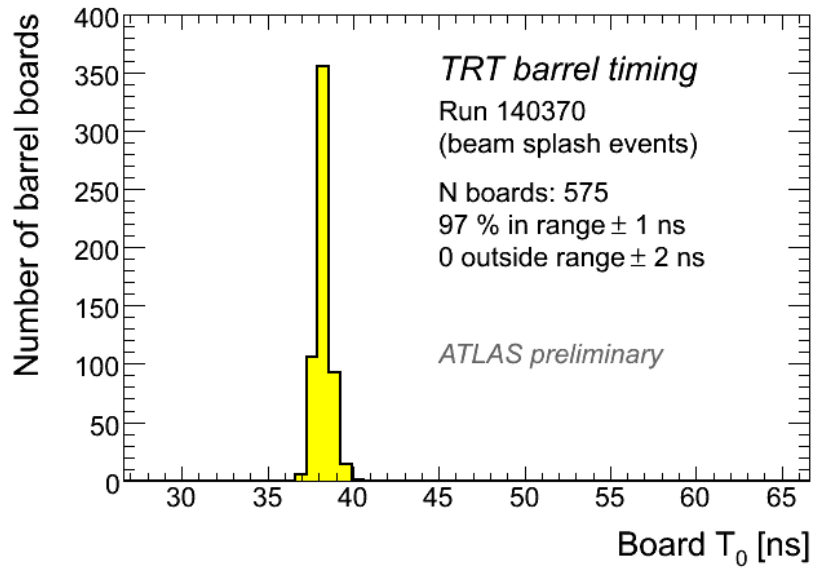

(a)

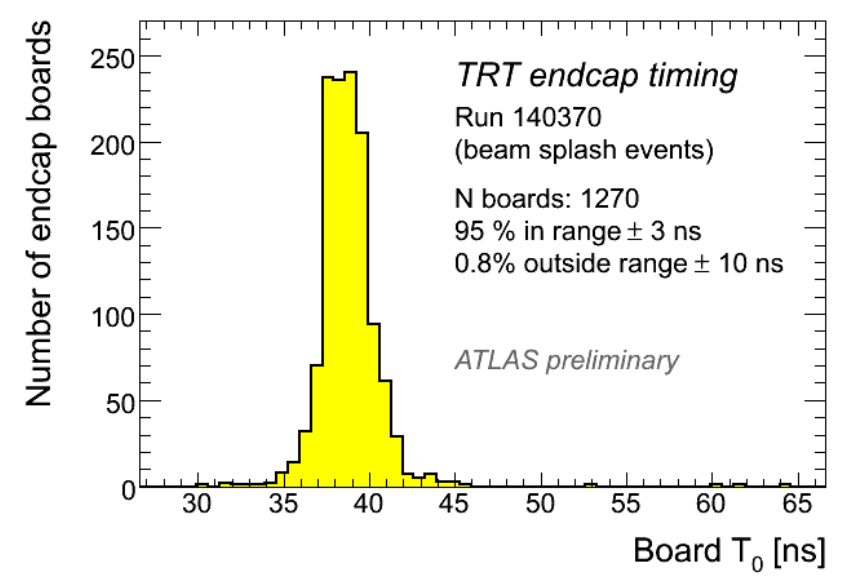

(b)

Figure 1: The TRT readout timing measured in 2009 beam splash data for the barrel (a) and end-caps (b). the drift distance calculated from each reconstructed track as a ${ }^{88}$ function of the drift time measured in the front end electronics 89 ("RT relation"), for all straws in the barrel. The solid line is 90 a fit to this data and matches well with a fit to cosmics data ${ }^{91}$ (dashed). This parametrization is used to convert drift time to 92 drift distance as input to track reconstruction algorithms.

\section{Active Readout Fraction}

An estimate of the active readout fraction can be obtained 98 from noise data. Results from test beam measurements indi- 99 cate [3] that a straw noise occupancy of $2 \%$ is equivalent to $a_{100}$ tracking threshold of $\sim 250 \mathrm{eV}$. Figure 3 shows the noise oc-101 cupancy for all readout channels after the tracking thresholds 102 have been equalized to this level. While the majority of read-103 out channels shows a good uniformity at $2 \%$ occupancy, outlier 104 channels with more ("noisy") or less ("dead") occupancy are 105 used to determine the fraction of channels that is unusable for ${ }_{106}$ track reconstruction to $\sim 2 \%$ with approximately the same frac-107 tion for barrel and both end-caps together. About $85 \%$ of the 108

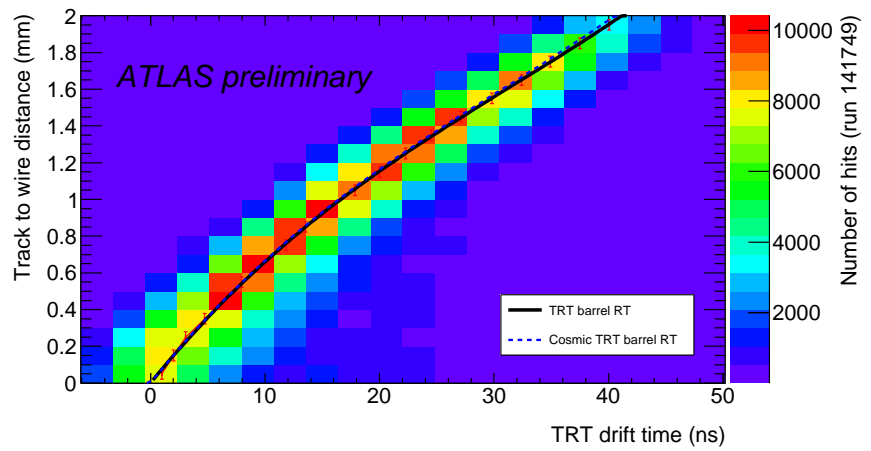

Figure 2: The RT dependency for the barrel from LHC collision events. The dashed line shows a fit to the RT dependency from cosmics data, and matches with a fit (solid) to the collision data. unusable barrel channels were already found before the detector was installed in the ATLAS cavern.

\section{Transition Radiation}

The TRT permits the discrimination of electrons from charged hadrons using transition radiation (TR). As the barrel straws are immersed in polypropylene fibers (interleaved foils in the end-caps), TR photons with an energy of more than $5 \mathrm{keV}$ can be produced when a charged particle with a Lorentz factor $\gamma>1000$ crosses the $\sim 30$ boundaries between the fibers and the active gas in the straw. Xe active gas has been chosen for its short absorption length for photons of this energy. As at this energy the photoelectric effect dominates, the energy of one absorbed TR photon is contained in a single straw. The front end electronics can separate straw signals from these high energy deposits and regular tracking hits using a separate "high threshold" (HT) discriminator set at $\sim 6 \mathrm{keV}$. Figure 4 a shows the probability that the HT is crossed per straw as a function of $\gamma$ for end-cap tracks from collision events. Clearly visible is the TR onset at $\gamma>1000$. The non-zero fraction for charged tracks comes from the probability to have a $\frac{d E}{d x}$ above the HT. The electrons in this sample have been selected from photons that convert before they enter the TRT, as shown in an example event in Fig. 4b. One of the tracks is required to pass tight electron selection criteria while the HT probability is measured on the other. The HT is calibrated such that it is accurate to $1.2 \%$ to optimize pion-electron separation. The current precision is close to this value. 


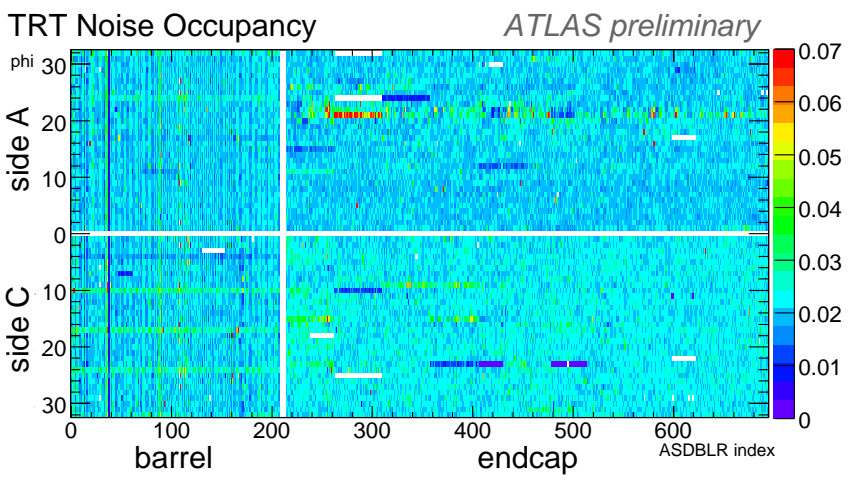

Figure 3: The noise occupancy for barrel and both end-caps for groups of 8 straws ("ASDBLR index"). While the plot shows a good uniformity at $2 \%$, outliers are used to determine the fraction of readout channels that is unusable for track reconstruction to $\sim 2 \%$.

\section{Front End Electronics and TRT Fast-OR Trigger}

When the LHC incident in Sept. 2008 promised an extension of the commissioning period using cosmics, the decision has been taken to finalize the implementation of a TRT trigger. It allowed the TRT to collect tracks from cosmics independent ${ }^{140}$ from other subsystems, with rates in both barrel and endcap that were significantly higher than what other triggers had been able to produce.

A "Fast-OR" circuit on the DTMROC [4] permits sending a trigger signal to the TTC board if it receives a discriminated straw signal from any of the 16 associated readout channels. The TTC back-end receives trigger signals from groups of 1015 DTMROCs over a communication line usually used for configuration and sense data transmission. Independent logic circuits on each of the 16 TTC boards in the barrel system can generate a trigger signal if the number of communication lines that carry a signal within a clock cycle ( $25 \mathrm{~ns}$ ) exceeds a configurable number.

To ensure a high fraction of hits on track ( $\sim$ a third) as well as very low noise, the trigger electronics on the DTMROC was configured to generate a signal from the high threshold that was lowered to minimum ionizing particle levels. Each TTC board ${ }_{142}$ was configured to send a trigger signal if at least 4 communication lines carry a signal. As minor disadvantages, this configu-143 ration makes TR calibration difficult and is not compatible with ${ }_{144}$ configuration or sense data transmission.

Figure 5 shows the trigger jitter from cosmic tracks that tra-146 verse the barrel. As more than $90 \%$ of the triggers arrive in one ${ }_{147}$ clock cycle, the TRT Fast-OR soon became a reference for the ${ }_{148}$ timing-in of other ATLAS triggers. It helped improve the muon ${ }_{149}$ system (RPC) trigger timing as well as inner detector readout ${ }_{150}$

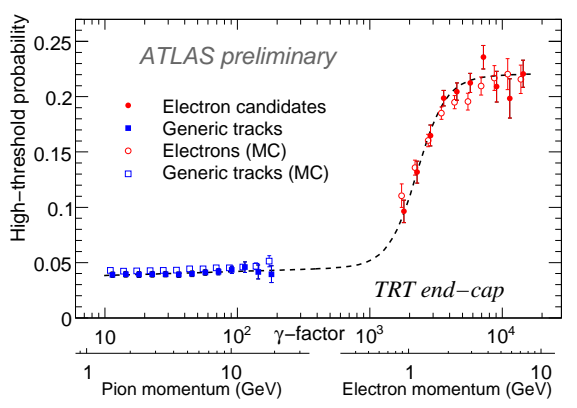

(a) (b)

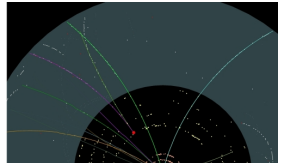

Figure 4: (a) Fraction of straw hits on track that have a HT signal as a function of $\gamma=\frac{E}{m}$ for the TRT end-caps as measured in collision events from Dec. 2009. The electrons have been selected from photon conversion as shown in an example event display in (b) where the photon converts in the Silicon Semiconductor Tracker (SCT).

timing. The trigger rate is $\sim 10 \mathrm{~Hz}$ and the efficiency of collecting cosmics tracks is $\sim 75 \%$.

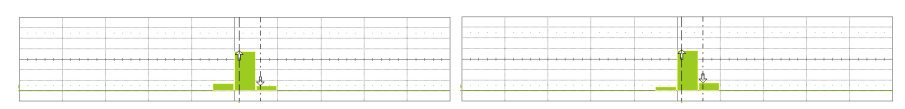

(a)

(b)

Figure 5: The jitter of trigger pulses from the A side of the TRT in (a) for tracks that crossed both barrel sides, with the trigger pulse from the $\mathrm{C}$ side as a reference point at 0 (centered). (b) The same for barrel $\mathrm{C}$ side with the A side as a reference. The histogram shows the data from 1000 accumulated triggers in units of ATLAS clock cycles ( $25 \mathrm{~ns}$ ). The jitter is estimated to be $8.75 \mathrm{~ns}$. See [4] for more details.

\section{Position Resolution}

A high position resolution of tracks that traverse the full inner detector is important for a good transverse momentum measurement of the track. Figure 6 shows the resolution for barrel tracks from collision events of Dec. 2009, after the data has been calibrated for alignment, RT relation and readout timing. The resolution of $165 \mu \mathrm{m}$, a value similar to what has been produced on cosmics data, is in agreement with the technical design report [2]. 


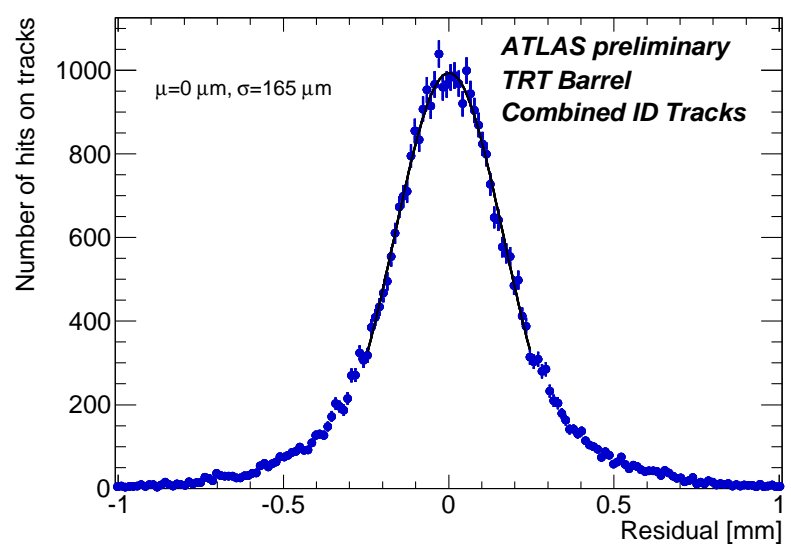

Figure 6: The position resolution in the barrel using tracks from collision events taken in Dec. 2009. The resolution of $165 \mu \mathrm{m}$ is in agreement with the technical design report.

\section{Conclusion}

The TRT commissioning process with cosmics and first collisions has been very successful. Trigger capabilities that have not been part of the original design have been implemented and used throughout the commissioning process to calibrate both the TRT and other ATLAS subsystems. The TRT performance in the barrel is within expectations of the technical design report. While many analyses, such as a material mapping of the inner detector, already make use of TRT data, the TRT is in an excellent shape to produce more collision data in the coming months.

\section{References}

[1] The ATLAS TRT Collaboration, "The ATLAS TRT Electronics," E. Abat et al., 2008 JINST 3 P06007; The ATLAS TRT Collaboration, "The ATLAS TRT barrel detector," E Abat et al., 2008 JINST 3 P02014; The ATLAS TRT Collaboration, "The ATLAS TRT end-cap detectors," E Abat et al., 2008 JINST 3 P10003.

[2] The ATLAS Collaboration, "The ATLAS Experiment at the CERN Large Hadron Collider," 2008 JINST 3 S08003.

[3] P. Cwetanski, "Straw Performance Studies and Quality Assurance for the ATLAS Transition Radiation Tracker," CERN-THESIS-2006-025.

[4] S. Fratina et al., "The TRT Fast-OR Trigger," ATL-INDET-PUB-2009002. 